\title{
Régimen histórico de incendios y su relación con el clima en un bosque de Pinus hartwegii al norte del estado de Puebla, México
}

\author{
Historical fire regime and its relationship with climate in a forest \\ of Pinus hartwegii to the north of Puebla State, Mexico
}

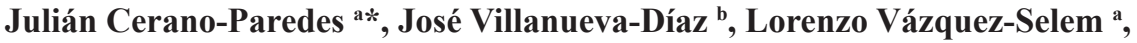 \\ Rosalinda Cervantes-Martínez $^{\text {b }}$, Gerardo Esquivel-Arriaga ${ }^{\text {b }}$, Vidal Guerra-de la Cruz ${ }^{\mathrm{c}}$, Pete Z Fulé ${ }^{\mathrm{d}}$ \\ * Autor de correspondencia: a Universidad Nacional Autónoma de México, Instituto de Geografía, \\ Ciudad Universitaria, Coyoacán, México D. F. México, C.P. 04510, cerano.julian@gmail.com \\ ${ }^{\mathrm{b}}$ INIFAP CENID-RASPA, km 6,5 Margen Derecha del Canal Sacramento, Gómez Palacio, Durango. México. \\ ' INIFAP-Tlaxcala, km 2,5 Carretera Tlaxcala-Chiautempan, Tlaxcala, México. \\ ${ }^{\mathrm{d}}$ Northern Arizona University, School of Forestry, Flagstaff, Arizona, USA.
}

\begin{abstract}
SUMMARY
The Pinus hartwegii forest in cooperative lands Rinconada, Chignahuapan, represents the region of highest risk of forest fires in the state of Puebla. In order to reconstruct the history of fires and determine their historical association with climate, we collected 43 cross-sections with fire scars and analyzed them by dendrochronological techniques. Two hundred and thirty five fire scars were dated in 40 cross-sections. The fire history was reconstructed for the period 1876-2011 (136 years). Most (91.7 \%) fires occurred during spring and $8.3 \%$ in summer. The Mean Fire Interval (MFI) and Weibull Median Probability Interval (WMPI) for all the scars were 5.6 and 5.0 years, respectively. Large or extensive fires occurred when fire scars were recorded in $25 \%$ of samples or more with MFI and WMPI values of 9.2 and 8.5 years, respectively. On this site, fires have continued to the present, but after 1988 a significant drop in fire frequency was noted. A positive association between lower precipitation (drought) and fire occurrence was determined. Similarly, negative values of NIÑO 3 and PDSI (dry conditions), associated with drought conditions, have a positive influence on the modulation of the historical frequency of fires in this region of central Mexico.
\end{abstract}

Key words: fire scars, precipitation, El Niño Southern Oscillation, Palmer drought severity index.

\section{RESUMEN}

El bosque de Pinus hartwegii en el ejido Rinconada, municipio de Chignahuapan, representa la región de mayor incidencia de incendios forestales en el estado de Puebla. Con el objetivo de reconstruir la historia del fuego y determinar su asociación histórica con el clima, se tomaron 43 muestras con cicatrices de incendios y se analizaron con base en técnicas dendrocronológicas; de 40 muestras, se lograron datar 235 cicatrices de incendios. Se reconstruyó la historia del fuego para el período 1876-2011 (136 años). El 91,7 \% de los incendios se registró en la estación de primavera y el 8,3\% en verano. El intervalo medio de incendios (MFI, por sus siglas en inglés) y el intervalo de la mediana de probabilidad de Weibull (WMPI, por sus siglas en inglés) al considerar todas las cicatrices fue de 5,6 y 5,0 años, respectivamente; mientras que para los incendios más grandes o extensos cuyas cicatrices se registraron en el $25 \%$ de las muestras o más, los valores de MFI y WMPI fueron de 9,2 y 8,5 años, respectivamente. La presencia de incendios fue continua hasta la primera década del siglo XXI; sin embargo, posterior a 1988 se observó una disminución en su frecuencia. Se determinó una relación positiva entre la disminución de la precipitación (sequía) y la ocurrencia de incendios. De igual manera, valores negativos de NIÑO 3 y PDSI, asociados a condiciones de sequía, han influido positivamente en la modulación de la frecuencia histórica de incendios en esta región del centro de México.

Palabaras clave: cicatrices de fuego, precipitación, El Niño Oscilación del Sur, índice de severidad de sequía de Palmer.

\section{INTRODUCCIÓN}

La variabilidad climática es un factor determinante en la variación histórica de la actividad de incendios forestales (Heyerdahl y Alvarado 2003, Fulé et al. 2005). En las últimas décadas el incremento de la actividad de incendios se ha atribuido al cambio climático global y se pronostica un aumento alredor del mundo (Westerling et al. 2006). La fuerte vinculación entre el fuego y el clima, ponen en manifiesto la relevancia de conocer la relación clima-incendios en distintos tipos de ecosistemas forestales para el diseño de prácticas de manejo del fuego y de adaptación al cambio climático. 
Los incendios forestales requieren biomasa, condiciones atmosféricas propicias para la propagación del fuego (es decir, ambiente seco, caliente y vientos fuertes) y una fuente de ignición (Krawchuk y Moritz 2011). El clima puede afectar estos tres factores en conjunto de diferentes maneras y en múltiples escalas de tiempo. Los regímenes del fuego en los ecosistemas han cambiado a través de la historia, especialmente en relación con los cambios en el clima y las actividades humanas (Pausas y Fernández-Muñoz 2011).

Para la parte norte de México, se han desarrollado diversos estudios de reconstrucción de la historia del fuego, los cuales han permitido determinar la influencia de la variabilidad climática en la frecuencia de incendios (Heyerdahl y Alvarado 2003, Fulé et al. 2005, Cerano-Paredes et al. 2010, Yocom et al. 2010). Así mismo, se han generado diferentes estudios con resultados que ponen de manifiesto el efecto de la actividad humana en el cambio de los regímenes históricos del fuego durante diferentes períodos del siglo XX (Heyerdahl y Alvarado 2003, Fulé et al. 2005, Cerano-Paredes et al. 2010, Yocom et al. 2010).

Para el centro del país, este tipo de estudios son escasos; actualmente solo se ha publicado una cronología de incendios para los pinares de alta montaña del Pico de Orizaba, Veracruz/Puebla (Yocom y Fulé 2012) y un trabajo más en los bosques de Pinus douglasiana Martínez en la Reserva de la Biosfera Sierra de Manatlán, Jalisco (Cerano-Paredes et al. 2015).

La comunidad de Pinus hartwegii Lindley presenta un régimen de incendios superficiales, frecuentes y de baja intensidad, se estima que la frecuencia promedio de incendios en estas comunidades no es superior a los 10 años, y probablemente la media está en torno a cinco años (Rodríguez-Trejo 2001). A la fecha no se ha generado una red de investigaciones fundamentada en técnicas dendrocronológicas para estudiar el régimen de fuego en este ecosistema, uno de los primero trabajos para este tipo de bosque en el Pico de Orizaba, reporta regímenes de 3 a 9 años (Yocom y Fulé 2012).

Para generar mayor conocimiento sobre los regímenes históricos de fuego en los bosques del centro de México, que permita evaluar el comportamiento de esta perturbación y seleccionar las estrategias más apropiadas para su manejo, se consideró el bosque de $P$. hartwegii enclavado en la Sierra Norte de Puebla, en el ejido Rinconada, municipio de Chignahuapan, región de mayor incidencia de incendios forestales en el estado de Puebla (Carrillo et al. 2012). Ante esto se plantearon los siguientes objetivos: 1) reconstruir la historia del fuego, 2) determinar si en las últimas décadas existe un cambio en la frecuencia de incendios y 3) analizar la relación entre las condiciones de clima (sequías) y la frecuencia histórica de incendios.

Se plantearon las siguientes hipótesis: 1) los regímenes del fuego han cambiado en las últimas décadas del siglo XX y este cambio está asociado principalmente a la influencia humana y 2) las condiciones extremas del clima, tales como sequías, han influido históricamente en la frecuencia de incendios.

\section{MÉTODOS}

Área de estudio. Se localiza en el ejido Rinconada, municipio de Chignahuapan, al norte del estado de Puebla, en los límites con el estado de Tlaxcala, entre los $19^{\circ} 42^{\prime}$ $06^{\prime \prime}$ $19^{\circ} 42^{\prime} 30^{\prime \prime} \mathrm{N}$ y $98^{\circ} 13^{\prime} 42^{\prime \prime}-98^{\circ} 14^{\prime} 06^{\prime \prime} \mathrm{O}$ (figura 1 ). El bosque de $P$. hartwegii en esta zona se distribuye de 2.800 a 3.000 m s.n.m.

El clima es del tipo $C b w$, templado con verano fresco y régimen de lluvias de verano (García 1987), temperaturas promedio mensuales que varían de $10^{\circ} \mathrm{C}$ la mínima en los meses más fríos (invierno) a $16^{\circ} \mathrm{C}$ en los meses más cálidos (verano), con máximas entre $20^{\circ} \mathrm{C}$ y $24^{\circ} \mathrm{C}$. La precipitación promedio anual es de $710 \mathrm{~mm}$, con el mayor porcentaje presentándose en el verano y una mínima cantidad en las estaciones de invierno-primavera (figura 2), registros promedio (1961-2000) de la estación El Rosario, localizada a tres kilometros del área de estudio y a $2.700 \mathrm{~m}$ s.n.m. (IMTA 2009). La estación meteorológica de referencia (El Rosario) representa el límite inferior de la distribución del bosque de $P$. hartwegii, la temperatura media en el límite superior (3.000 m s.n.m.) debe ser alrededor dos grados inferior.

La zona presenta una cubierta vegetal propia del clima templado frío. Las especies arbóreas más importantes, tanto por su abundancía como por su valor económico son los géneros de pino (Pinus patula Schlecht et Cham, P. teocote Schiede ex Schltdl, $P$. hartwegii), oyamel (Abies religiosa (H.B.K) Schlecht et Cham), encino (Quercus spp.), aile (Alnus sp.) y madroño (Arbutus xalapensis Kunth). En la parte baja de la zona boscosa, domina un bosque de coníferas con una mezcla de especies de $P$. patula, P. teocote, Quercus spp. y Arbutus xalapensis; mientras que en la parte alta (2.900-3.000 m s.n.m) se presenta un bosque con dominancia de $P$. hartwegii y $A$. religiosa asociados con $P$. patula, Arbutus xalapensis, Quercus spp. y Alnus sp.

Métodos de campo. En la parte alta de la zona boscosa en una superficie aproximada de 70 ha y un gradiente de 2.900-3.000 m s.n.m., se seleccionaron de forma dirigida árboles de $P$. hartwegii con cicatrices de incendios. Se removieron secciones parciales de árboles vivos, muertos en pie, caídos y tocones, con una motosierra (figura 3). Se consideraron los individuos con el mayor número de cicatrices (registros de incendios), lo mejor preservados en el caso de arbolado muerto y lo más longevos posible.

Métodos de laboratorio. En el laboratorio, las muestras fueron montadas y pulidas con lijas de grano 40 a 1.200 para lograr una superficie uniforme y apreciar de manera clara el xilema bajo microscopio (figura 3B). Las muestras se fecharon con base en una cronología maestra de anillo total de P. patula generada para la región (Quezada 2013), que presentó una correlacion entre series de 0,61 


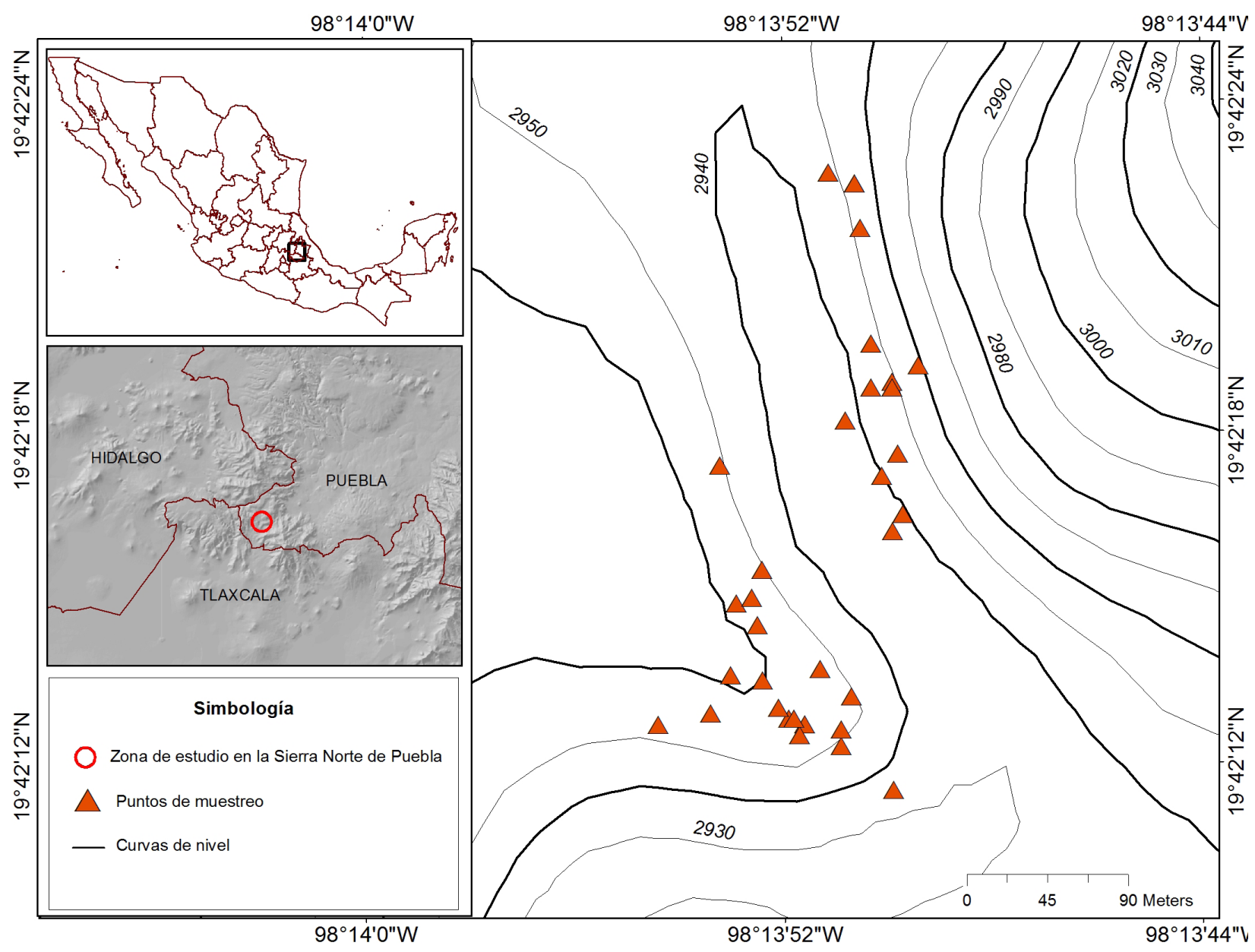

Figura 1. Localización geográfica del área de estudio, bosque de Pinus hartwegii en el ejido Rinconada, municipio de Chignahuapan, Puebla. Los triángulos representan los diferentes puntos muestreados.

Geographical location of the study area, Pinus hartwegii forest in the cooperative lands Rinconada, municipality of Chignahuapan, Puebla. The triangles represent different points sampled.

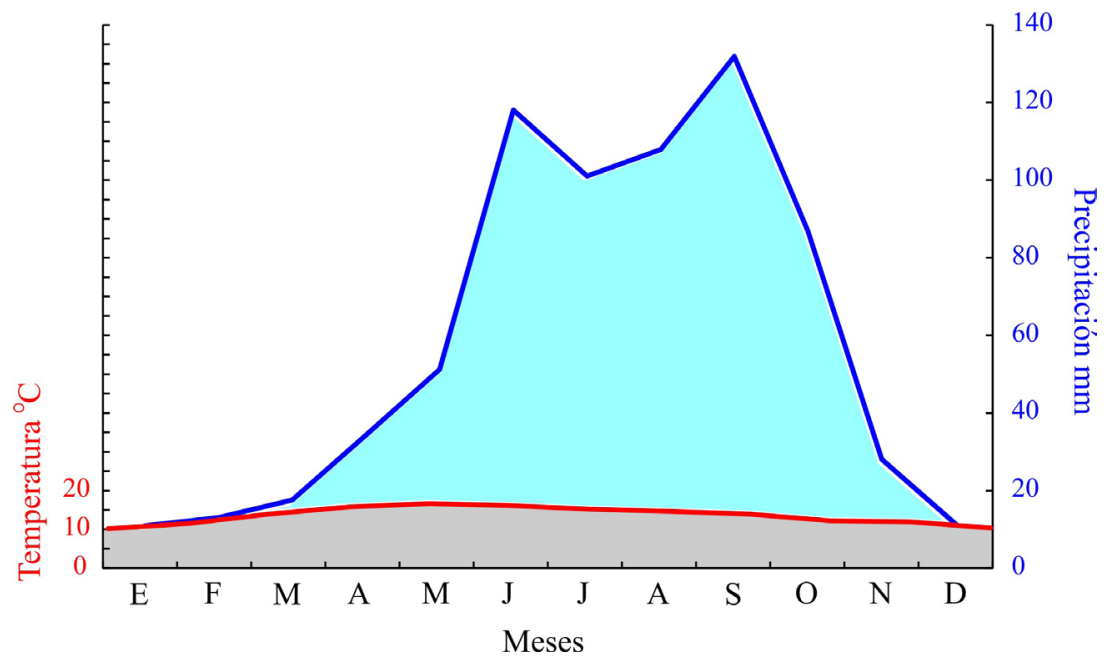

Figura 2. Climograma de la precipitación y temperatura media mensual del período 1961-2000 para la estación meteorológica El Rosario. El área muestra un régimen de lluvias de verano y una escasa precipitación durante el período invierno-primavera (IMTA 2009).

Climograph, monthly precipitation and average temperature for the period 1961-2000 for the weather station El Rosario. The area shows a summer rainfall and low rainfall during the winter-spring period (IMTA 2009). 


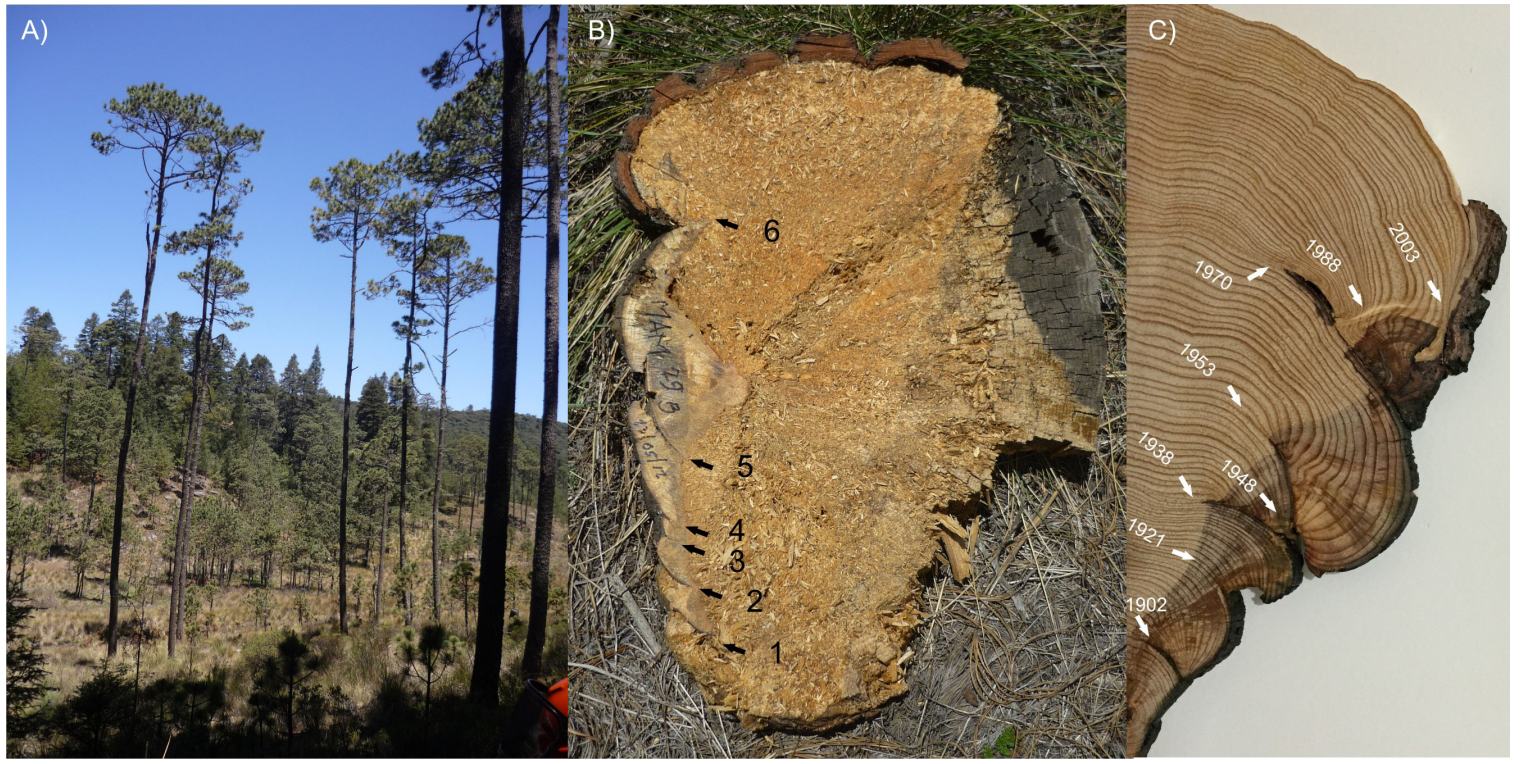

Figura 3. (A) Bosque de Pinus hartwegii al norte del estado de Puebla. (B) Ejemplar tomado de un tocón que muestra evidencia de registros de incendios (seis cicatrices). Este tipo de muestras permiten extender en el tiempo la historia de incendios y (C) es una sección parcial de un árbol vivo de Pinus hartwegii posterior a su preparación y fechado. La muestra presenta registros históricos de ocho cicatrices de incendios.

(A) Pinus hartwegii forest in the north of Puebla State. (B) Specimen taken from a stump showing evidence of fire logs (six scars). Such samples allow you to extend in time the fire history and $(\mathrm{C})$ is a partial section of a live tree Pinus hartwegii after their preparation and dated. The sample presents historical records of eight fire-scars.

$(P<0,01 ; \mathrm{n}=97)$, una longitud de 107 años y explica el $61 \%(P<0,001)$ de la variabilidad de la precipitación invierno-primavera. Se determinó el año exacto de formación de cada uno de los crecimientos anuales, mediante la comparación de los patrones de crecimiento (Stokes y Smiley 1968).

Después del fechado, el ancho de anillo de las muestras se midió con un sistema de medición Velmex de precisión $0,001 \mathrm{~mm}$. La calidad del fechado se verificó con el programa COFECHA (Holmes 1983). Las cicatrices de los incendios se emplearon como evidencia de incendios, identificados como una discontinuidad en las células a lo largo del límite del anillo de crecimiento, donde el cambium murió, seguido por una supresión y deformación de los crecimientos (Dieterich y Swetnam 1984).

La estación de ocurrencia de los incendios se estimó con base en la posición relativa de cada una de las cicatrices de incendio en el anillo anual, considerando las siguientes categorías: EE (inicio de la madera temprana), ME (mitad de la madera temprana), LE (final de la madera temprana), L (madera tardía) y D (dormancia o límite del anillo) (Dieterich y Swetnam 1984). Las cicatrices determinadas en la estación de dormancia se asignaron al inicio de la madera temprana del siguiente año (incendios de primavera) (Heyerdahl y Alvarado 2003, Swetnam y Baisan 2003, Yocom y Fulé 2012). Las diferentes categorías se agruparon en dos períodos: 1) primavera $(\mathrm{D}+\mathrm{EE})$ y 2 ) verano (ME + LE + L) (Grissino-Mayer 2001).
Análisis de datos. La base de datos de historia del fuego se analizó con el programa FHX2, versión 3.2 (GrissinoMayer 2001). El análisis se corrió a partir del primer año con un adecuado tamaño de muestra (1902), definido por el primer año de incendio registrado en el $10 \%$ o más del número total de muestras (Grissino-Mayer et al. 1994) y finalizó en el año 2011.

Los intervalos medios de frecuencia y recurrencia de incendios se analizaron estadísticamente con tres diferentes filtros: (1) todas las cicatrices de incendio, (2) cicatrices presentes en $\geq 10 \%$ de las muestras y (3) cicatrices de registradas en $\geq 25 \%$ del total de las muestras. El filtro de $25 \%$, fue utilizado como un estimador de la frecuencia de incendios relativamente más extensos e intensos (Swetnam y Baisan 2003).

Para cada uno de los filtros, se determinaron los siguientes estadísticos descriptivos: intervalo medio de frecuencia de incendios (MFI, por sus siglas en inglés), número de intervalos, intervalo mínimo y máximo, y el intervalo mediana de probabilidad de Weibull (WMPI, por sus siglas en inglés). Esta última es una medida de distribución central, empleada para modelar la distribución asimétrica de los intervalos de incendios y para expresar en términos probabilísticos los intervalos de recurrencia (Grissino-Mayer et al. 1994).

Relación clima-incendios. Para analizar las condiciones climáticas y su relación con la frecuencia de incendios, se 
empleó el análisis de sobreposición de época (SEA, por sus siglas en inglés) del programa FHX2 versión 3.2 (Grissino-Mayer 2001). Como proxy del clima, se emplearon tres variables: 1) una reconstrucción de precipitación local (invierno-primavera) generada con base en anillos de crecimiento de Pinus patula para el período 1905-2011 (Quezada 2013), 2) el índice invernal (diciembre-febrero) NIÑO 3 de El Niño Oscilación del Sur (ENSO, por sus siglas en inglés) (Cook 2000a) y 3) el índice de severidad de sequía de Palmer (PDSI, por sus siglas en inglés) (Cook 2000b). Cada una de estas tres variables se analizó por separado con la reconstrucción de la historia del fuego empleando la rutina SEA, comparando las condiciones durante el año del incendio, cinco años previos, y dos años posteriores al mismo. Para evaluar la significancia estadística del análisis SEA, los intervalos de confianza (95, 99 y 99,9 \%) se calcularon utilizando la distribución bootstrapped de los datos climáticos con 1.000 repeticiones. Finalmente, se utilizó el Atlas de Sequía para México (MXDA, por sus siglas en ingles) (Stahle et al. 2016) para analizar las condiciones climáticas de años donde los incendios afectaron la mayoría de los árboles del sitio.

\section{RESULTADOS}

Se colectaron un total de 43 muestras con cicatrices de incendios ( 25 de árboles vivos y 18 de muertos) y se logró fechar 235 cicatrices al año exacto de su formación correspondientes a 40 muestras (93\%); se descartaron tres muestras ( $7 \%$ ), cuyo grado de deterioro impidió su fechado. El mayor número de cicatrices se observó en el año 1970 (39), el 98 \% de los árboles registraron este evento que representó el incendio más extenso de la zona durante el siglo XX (figura 4).

Estacionalidad de los incendios. La posición de la cicatriz del incendio dentro del anillo de crecimiento que define la estación del año en que se registró el incendio fue determinada para el 92,2 \% de las muestras; la posición de la cicatriz más común fue al inicio de la madera temprana $(91,7 \%)$, y la menos común en la madera tardía (0 \%). El 91,7 \% de los incendios se registraron en primavera (estación seca) y el $8,3 \%$ en verano (cuadro 1 ).

Frecuencia de incendios. Se logró reconstruir la historia del fuego para los últimos 136 años que comprende el período de 1876 a 2011. El incendio más antiguo se dató en el año 1890 y la cicatriz más reciente ocurrió en el año 2003 (figura 4). Para todas las cicatrices de incendios, se determinó un MFI de 5,6 años y un WMPI de 5,0 años. Al considerar las cicatrices registradas en el $10 \%$ las muestras o más, se determinó un MFI y un WMPI de 7,2 y 6,1 años, respectivamente (cuadro 2). Para los incendios más grandes o extensos definidos por las cicatrices datadas en el $25 \%$ de las muestras o más, los valores de MFI y WMPI fueron de 9,2 y 8,5 años, respectivamente (cuadro 2).

Cuadro 1. Determinación de la estacionalidad de las cicatrices de incendios.

Determination of the seasonality of the fire scars.

\begin{tabular}{|c|c|c|c|c|c|c|c|c|c|c|}
\hline $\begin{array}{l}\text { Sitio } \\
\text { CHIG }\end{array}$ & Total & $\begin{array}{c}\text { Estación } \\
\text { determinada }\end{array}$ & $\begin{array}{l}\text { Estación no } \\
\text { determinada }\end{array}$ & $\mathrm{D}$ & $\mathrm{EE}$ & $\mathrm{ME}$ & LE & $\mathrm{L}$ & $\begin{array}{l}\text { Incendios } \\
\text { primavera }^{\mathrm{A}}\end{array}$ & $\begin{array}{c}\text { Incendios } \\
\text { verano }^{\mathrm{B}}\end{array}$ \\
\hline $\begin{array}{l}\text { Número } \\
\text { cicatrices }\end{array}$ & 234 & 216 & 18 & 0 & 198 & 17 & 1 & 0 & 198 & 18 \\
\hline Porcentaje & 100 & 92,3 & 7,7 & 0 & 91,7 & 7,9 & 0,5 & 0 & 91,7 & 8,3 \\
\hline
\end{tabular}

Nota: La definición de la estacionalidad de los incendios para esta región del centro de México, fue con base en la metodología de Grissino-Mayer (2001). ${ }^{A}$ Dormancia + inicio de madera temprana $(\mathrm{D}+\mathrm{EE}) .{ }^{\mathrm{B}}$ Mitad de la madera temprana + final de la madera temprana + madera tardía $(\mathrm{ME}+\mathrm{LE}+\mathrm{L})$.

Cuadro 2. Intervalos de frecuencia de los incendios para el bosque de P. hartwegii del ejido Rinconada, Chignahuapan, Puebla. Período de análisis 1902-2011.

Fire frequency intervals in a forest of $P$. hartwegii in the cooperative lands Rinconada, Chignahuapan, Puebla.

\begin{tabular}{|c|c|c|c|c|c|}
\hline $\begin{array}{l}\text { Categoría } \\
\text { de análisis }\end{array}$ & $\begin{array}{c}\text { Número } \\
\text { de intervalos }\end{array}$ & $\begin{array}{l}\text { Intervalo medio } \\
\text { de frecuencia }\end{array}$ & Mínimo & Máximo & $\begin{array}{l}\text { Intervalo de la mediana de } \\
\text { la probabilidad de Weibull }\end{array}$ \\
\hline Todas las cicatrices & 18 & 5,6 & 2 & 19 & 5,0 \\
\hline $10 \%$ cicatrices & 14 & 7,2 & 2 & 19 & 6,1 \\
\hline $25 \%$ cicatrices & 11 & 9,2 & 2 & 19 & 8,5 \\
\hline
\end{tabular}




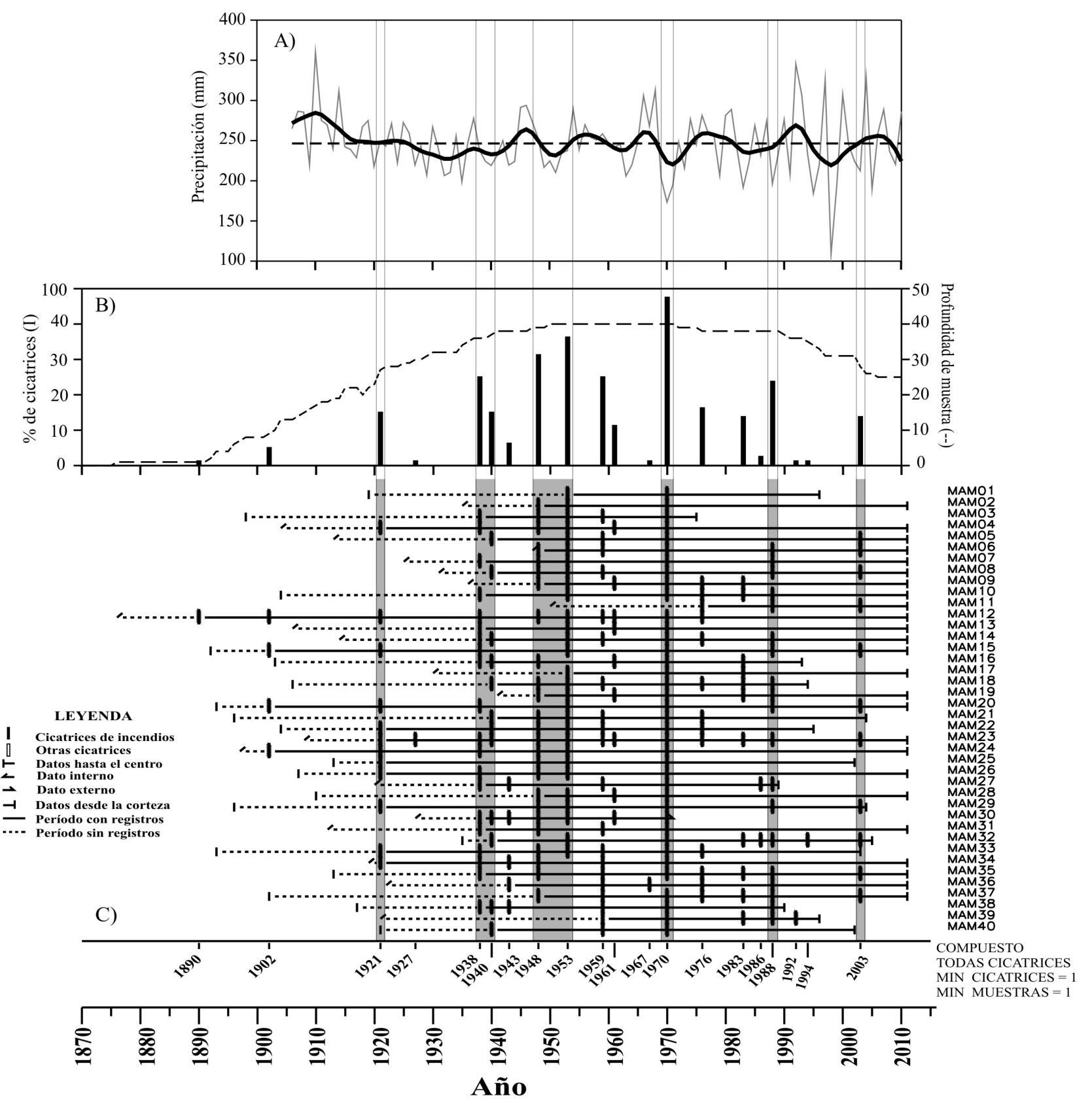

Figura 4. Historia del fuego en un bosque de Pinus hartwegii del ejido Rinconada, Chignahuapan, Puebla. (A) Indica la variabilidad de la precipitación para esta región en el último siglo (Quezada 2013), (B) muestra el número de eventos reconstruidos (20), porcentaje cicatrices por año y número de muestras por período y en (C) las líneas horizontales representan la longitud de cada uno de los árboles, punteadas antes del primer registro de incendio y sólidas para el período con registros. Las barras verticales en color negro representan las cicatrices de los incendios y, a medida que la línea se torna continua, indica mayor cantidad de árboles afectados y superficie. Los años en los que se registraron incendios se muestran en el eje secundario de las X.

Fire history in a forest of Pinus hartwegii cooperative lands Rinconada, Chignahuapan, Puebla. (A) Indicates the variability of precipitation for this region in the last century (Quezada 2013), (B) shows the number of reconstructed events (20), scars percentage per year and number of samples per period and $(\mathrm{C})$ horizontal lines represent the length of each of the trees, dotted before the first record of fire and sound for the period with records. The vertical bars in black represent the scars of fires and, as the line becomes continuous, it indicates superior amount of affected trees and/or surface. The years in which fires were reported are shown in the secondary $\mathrm{X}$-axis. 
Relación clima-incendios. El análisis de relación climaincendios SEA, indica que los incendios reconstruidos en el bosque de $P$. hartwegii se registraron durante años con valores de precipitación por debajo de la media y valores negativos de NIÑO 3 y PDSI (figura 5). En contraste, no se observaron incendios durante años con valores de precipitación por arriba de la media, ni valores positivos de PDSI y NIÑO 3, años que representan condiciones de mayor precipitación (figura 5). Las condiciones climáticas

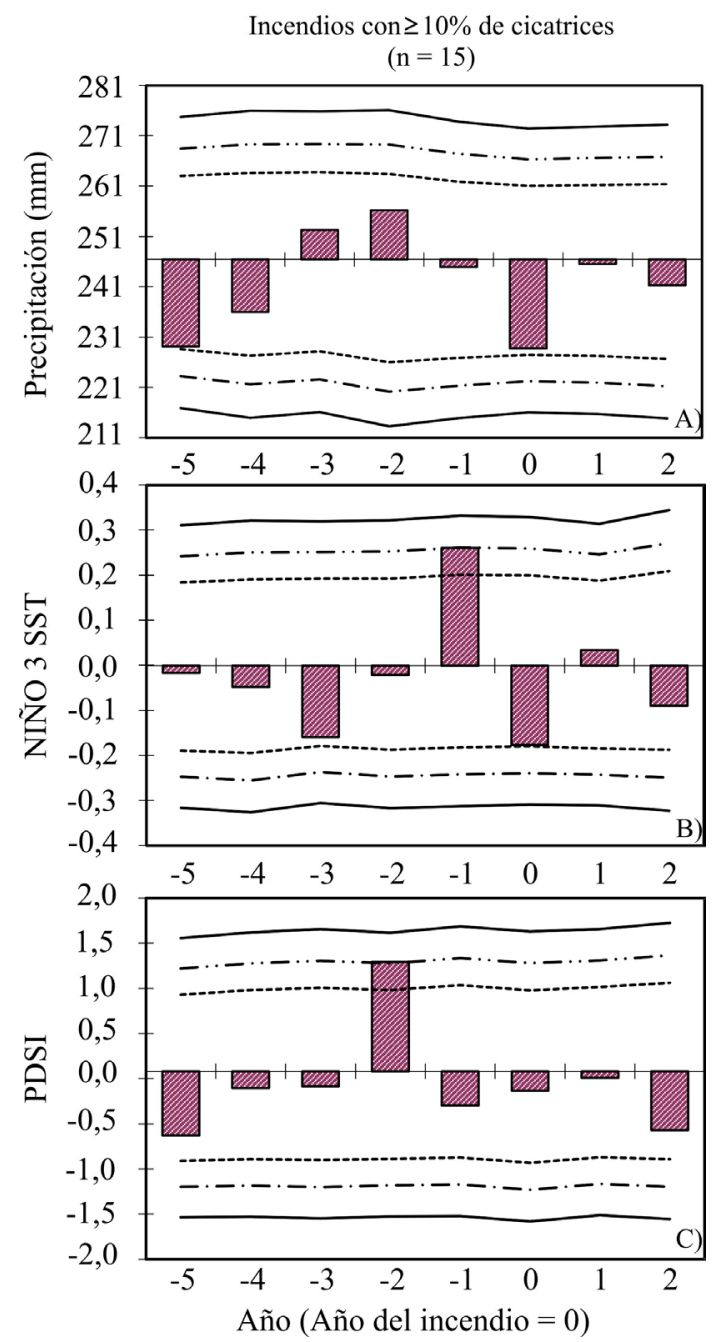

Figura 5. Análisis de sobreposición de época: muestra la relación entre la ocurrencia del incendio con el clima regional (reconstrucción de precipitación) (A), índices de NIÑO 3 (B) y PDSI (C), para todos los incendios registrados en $\geq 10 \%$ de las muestras. El año del incendio está indicado por el 0 , condiciones cinco años previos al incendio (valores negativos) y dos años posteriores al incendio (valores positivos). Las tres líneas superiores e inferiores representan los intervalos de confianza al $95 \%$, $99 \%$ y 99,9\%.

Superposed Epoch Analysis showing the relationship between the occurrences of fire with the regional climate (precipitation reconstruction) (A), NIÑO 3 (B) and PDSI (C) indices for all fires in $\geq 10 \%$ of the samples. The year of the fire is indicated by 0 , conditions five years before the fire (negative values) and two years after the fire (positive values). The upper and lower three lines represent confidence intervals $95 \%, 99 \%$ and $99.9 \%$. de NIÑO 3 y PDSI, 1 y 2 años previos al incendio, respectivamente, presentaron valores positivos (condiciones húmedas) de manera significativa $(P<0,01)$ (figura $5 \mathrm{~B}$ y $\mathrm{C}$ ).

La mayoría de los incendios reconstruidos para el período 1890-2011, se registraron ante condiciones de lluvia por debajo de la media (15 de 20 incendios; $75 \%$ ) (figura 6). Así mismo, más de la mitad de los incendios ocurrieron ante eventos NIÑO 3 (10 de 14 incendios, 71,4 \%) y PDSI (8 de 14 incendios, 57,1 \%) con valores negativos, condiciones de sequía para el centro de México (figura 6).

\section{DISCUSIÓN}

La reconstrucción de la variabilidad de la precipitación para esta región, muestra el año de 1970 como el segundo evento de sequía más intenso del siglo XX, solo superado por el año 1998 (Quezada 2013). Así mismo, registros climáticos observados del período 1961-2000 muestran este mismo comportamiento (IMTA 2009). La extensión y severidad de este evento, se puede atribuir a dos aspectos: uno, al intervalo de tiempo previo libre de incendios ( 9 años), condición que favoreció la acumulación de combustible tanto en cantidad como en continuidad, y dos, al efecto climático. Trenberth (1997) reporta un período de influencia del ENSO en su fase cálida El Niño de 19 meses, que se extiende de finales de 1968 a marzo de 1970, condición que representa sequía para el centro y sur de México (Magaña et al. 2003). Las condiciones de sequía un año previo al incendio (1970) como ocurrió en 1969 (figura 7), aunado al período libre de incendios que favoreció la acumulación de combustible, crearon el ambiente propicio para el desarrollo del incendio más extenso de los últimos 136 años. Situación similar se ha registrado para el norte de México, donde se han reconstruido incendios de alta intensidad, los cuales se han asociado a una elevada acumulación de combustible tras varios años libres de fuego y una posterior condición de sequía (Skinner et al. 2008).

Estacionalidad de los incendios. En el área de estudio las máximas temperaturas y la precipitación mínima se registran durante la estación de primavera (IMTA 2009) (figura 2), situación a la que se atribuye el elevado porcentaje de incendios en primavera $(91,7 \%)$. Los incendios en los bosques de $P$. hartwegii se registran en enero y continúa hasta mayo o junio (Rodríguez-Trejo 2001), lo cual se relaciona con el período seco de primavera.

Estudios desarrollados para el norte de México (Stephens et al. 2003, Heyerdahl y Alvarado 2003, Fulé et al. 2005, Cerano-Paredes et al. 2010) y para la región centro del país (Cerano-Paredes et al. 2015), reportan resultados similares sobre la estacionalidad de los incendios, coincidiendo un mayor número de cicatrices de incendios al inicio de la estación de crecimiento (madera temprana).

Frecuencia de incendios. Los regímenes reconstruidos corroboran lo reportado por Rodríguez-Trejo (2001) quien 

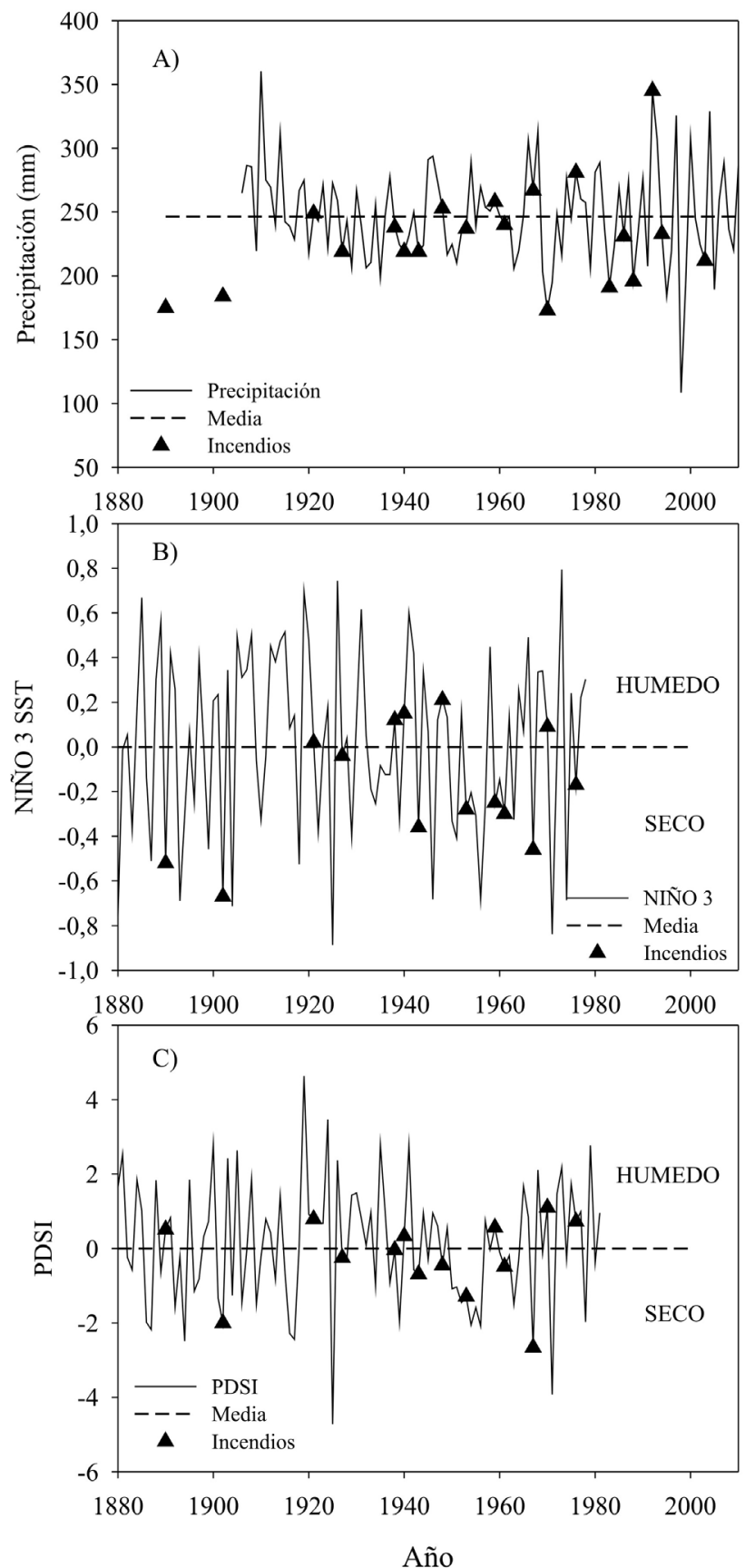

Figura 6. Condición anual del clima durante años de incendio (triángulos). Los tres diferentes gráficos de precipitación (A), NIÑO 3 (B) y PDSI (C) representan la reconstrucción de la variabilidad climática con base en anillos de crecimiento. (A) Muestra que la mayoría de los incendios reconstruidos para el período 1890-2011, se registraron ante condiciones de lluvia por debajo de la media y (B y C) que más de la mitad de los incendios ocurrieron ante eventos de NIÑO 3 y PDSI con valores negativos, condiciones de sequía.

Annual climate condition during fire years (triangles). The three different graphics precipitation (A), NIÑO 3 (B) and PDSI (C) represent the reconstruction of climate variability based on growth rings. (A) Shows that most fires reconstructed for the period 1890-2011 were recorded in conditions of precipitation below average and (B and $\mathrm{C}$ ) that over half of fires events occurred in NIÑO 3 and PDSI with negative values, drought conditions. indica que la frecuencia de incendios en los bosques de $P$. hartwegii no superan los 10 años, y presentan un promedio de cinco años, aproximadamente. Así mismo, se reafirman los regimenes reconstruidos con base en técnicas dendrocronológicas para los bosques de $P$. hartwegii del Pico de Orizaba en el centro de México por Yocom y Fulé (2012).

De un total 20 incendios reconstruidos durante los últimos 136 años, 14 (70 \%) presentan alta sincronía (figura 4), lo que indica la ocurrencia de eventos extensos (Fulé y Covington 1999, Swetnam y Baisan 2003). De finales del siglo XIX a principios del siglo XXI los incendios han sido frecuentes, trabajos desarrollados para el centro de México, han reconstruido una incidencia continua del fuego hasta los primeros años del siglo actual (Yocom y Fulé 2012, Cerano-Paredes et al. 2015). A diferencia de la zona centro del país, para la región norte, se ha documentado una exclusión del fuego a partir de 1930 (Yocom et al. 2010) o 1950 (Heyerdahl y Alvarado 2003, Fulé et al. 2005, Skinner et al. 2008).

Aunque existe una incidencia ininterrumpida del fuego para esta región, se ha detectado una disminución en su frecuencia posterior al año 1988. Durante el período de 1988 a 2003 (15 años), sólo se registraron dos incendios, en 1992 y 1994, registrados por un solo individuo, eventos muy localizados (figura 4). El año 1998 representa una de las temporadas de incendios más severas para el país, sin embargo; no existe evidencia de daños en el área. Esta disminución de la frecuencia de incendios en este periodo puede estar relacionada con una marcada influencia humana en el régimen local de incendios, lo cual soportaría nuestra primera hipótesis. El ejido presenta registros de aprovechamiento maderable regulado a partir de 1985, el cual también implica un control mayor en la ocurrencia de incendios (Morales 2005). Posterior al año 2003 (intervalo de 8 años), no se registró evidencia de incendios. Al considerar todas las cicatrices de incendios ocurridas durante el período 1988-2003, se estimó un MFI de 4,6 años, similar a los últimos 100 años; sin embargo, los incendios de mediana $(10 \%)$ y alta $(25 \%)$ intensidad indican un MFI de 15 años, superiores al MFI histórico (9,2 años). Es importante considerar, que la amplitud en los intervalos de la frecuencia de incendios propician un incremento en la acumulación de combustibles (hojas, pastos, arbustos y ramas), tanto en cantidad como en continuidad (Skinner et al. 2008), lo que puede favorecer la presencia de incendios más severos, en particular, cuando esto coincida con un año de sequía. Se recomienda desarrollar estudios que determinen las cargas de combustibles actuales que soporten la definición de áreas de mayor riesgo de incendios.

La información generada en este estudio resulta esencial para los encargados de manejo del bosque en esta región, quienes deberán reducir el peligro de incendios severos. El conocimiento de la historia del fuego fundamenta el diseño de estrategias para ejecutar acciones de manejo del fuego. El cambio en la frecuencia de incendios en las últi- 
A)
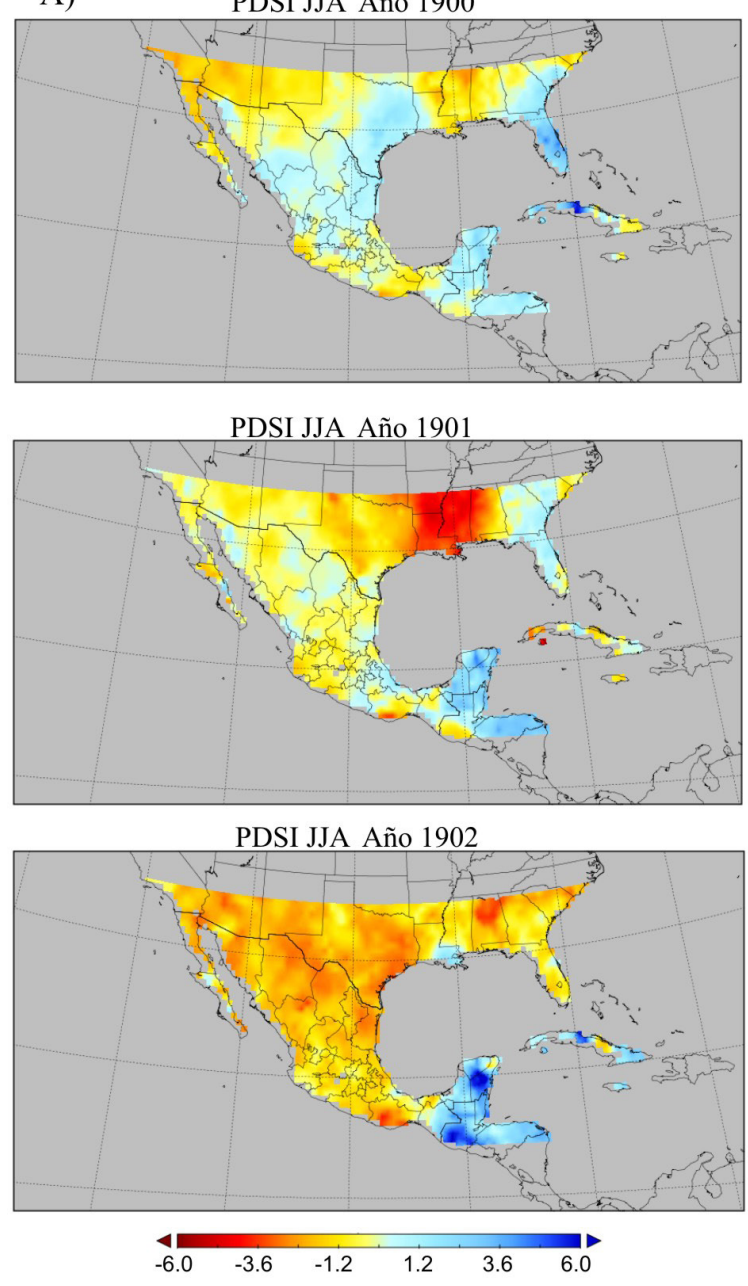

Proyección Gnomonic centrada sobre -97.5 E y $24.0 \mathrm{~N}$
B)

PDSI JJA Año 1968
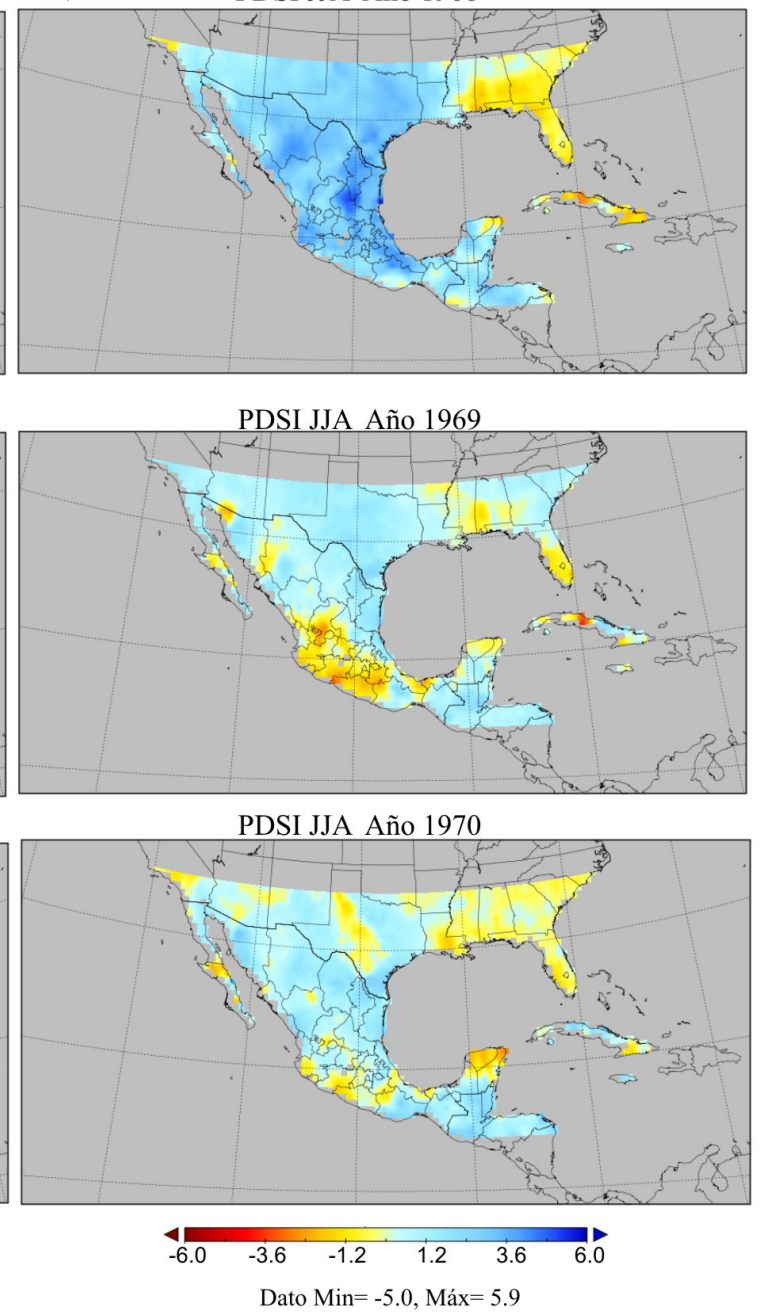

Figura 7. Mapas de sequía desarrollados utilizando el Atlas de Sequía para México (MXDA). Los mapas muestran las condiciones climáticas dominantes para dos años previos a los incendios (A) 1902 y (B) 1970. Ambos incendios son considerados extensos y afectaron el $50 \%$ y $95 \%$ del arbolado del sitio, respectivamente.

Drought maps developed using Drought Atlas of Mexico (MXDA). The maps show the prevailing climatic conditions for two years prior to the fire (A) 1902 and (B) 1970. Both fires are considered extensive and affected $50 \%$ and $95 \%$ woodland site, respectively.

mas décadas obedece posiblemente a una tendencia debido a una mayor efectividad en el control de incendios, por lo tanto, se debe mantener la frecuencia de incendios dentro de la amplitud de su variación histórica, con el objetivo de obtener beneficios ecológicos, reducir el riesgo de incendios severos y garantizar la permanencia de estos bosques.

Relación clima-incendios. El fenómeno ENSO, es un mecanismo de forzamiento climático que afecta a la precipitación y ocurrencia de incendios forestales en muchas partes del mundo (Heyerdahl y Alvarado 2003, Fulé et al. 2005, Skinner et al. 2008). En el sur de Estados Unidos y norte de México, los eventos cálidos (El Niño) se asocian con condiciones invernales húmedas y menor cantidad de incendios; a su vez, eventos fríos (La Niña) tienden a fa- vorecer inviernos secos y mayor ocurrencia de incendios (Heyerdahl y Alvarado 2003, Fulé et al. 2005). Un estudio reciente en la Sierra Madre Oriental, reporta un cambio temporal en la influencia de ENSO sobre los incendios, antes de 1830 La Niña fue asociada significativamente con años de incendio y posterior a 1830 esta asociación no fue significativa (Yocom et al. 2010). Para el sur de México, los efectos de este fenómeno muestran también un comportamiento dipolar aunque opuesto a lo observado en el norte, es decir, eventos El Niño propician inviernos secos que favorecen el desarrollo incendios y eventos La Niña se asocian a condiciones de mayor humedad (Magaña et al. 2003, Seager et al. 2009, Stahle et al. 2011).

Para el área de estudio se reconstruyó un total de 20 incendios (figura 4), de los cuales 18 se registran durante 
el siglo XX, 16 (89 \%) bajo la presencia de ENSO en su fase cálida (El Niño) y dos en condiciones de ENSO en su fase fría (La Niña) (Trenberth 1997, Gergis y Fowler 2009). Estos resultados corroboran la asociación entre ENSO y eventos de incendios para esta región, eventos El Niño son asociados significativamente $(P<0,05)$ con años de incendios. Lo anterior representa condiciones de sequía para el centro de México (Magaña et al. 2003, Seager et al. 2009, Stahle et al. 2011) y sustenta la segunda hipótesis de investigación, que establece que condiciones de sequía han influido en la frecuencia histórica del fuego en esta región del centro del país. A diferencia de este estudio, para los bosques de alta montaña de $P$. hartwegii en el Pico de Orizaba, Veracruz, no se encontró una asociación significativa entre El Niño y eventos de incendios, atribuible a la influencia antropogénica en el régimen de incendios (Yocom y Fulé 2012). Sin embargo, es importante destacar que eventos extremos de ENSO tanto en su fase cálida El Niño (figura 7A) como fría La Niña (figura 7B) impactaron ambas regiones, como ocurrió en los años de 1902 y 1968, respectivamente.

Los eventos La Niña de 1921 y 1943 (Gergis y Fowler 2009), que en teoría deberían haber impactado el norte de México, ocasionaron incendios importantes en este ecosistema de $P$. hartwegii del centro del país (figura 4). Por el contrario, los años El Niño de 1983 y 1998 (Trenberth 1997), que debieron haber propiciado condiciones secas para el sur del país, impactaron incluso la parte norte de México, donde se han reconstruido incendios de alta intensidad durante estos dos años (Yocom et al. 2010).

Las condiciones significativas $(P<0,01)$ de humedad para NIÑO 3 y PDSI uno y dos años previos a la ocurrencia del incendio, respectivamente, se asocian a una elevada producción de biomasa de plantas herbáceas y pastos, lo que aunado a una posterior disminución de la lluvia, origina condiciones propicias para el desarrollo de fuertes incendios (figura 7). Swetnam y Baisan (2003) indican que la propagación del fuego se ve favorecida cuando un año seco sigue a un año húmedo, probablemente por la alta producción de combustible fino. Las condiciones significativas de humedad para el centro del país uno (figura 5B) y dos años antes del incendio (figuras 7, 5A y 5C) han influido de manera significativa en la propagación del fuego.

Resultados similares sobre condiciones significativas $(P<0,05)$ de humedad antes de incendios intensos, se han reportado para diferentes bosques de coníferas en el norte de México (Stephens et al. 2003, Fulé et al. 2005, CeranoParedes et al. 2010, Yocom et al. 2010) y suroeste de los Estados Unidos (Swetnam y Baisan 2003).

\section{CONCLUSIONES}

La reconstrucción de la historia de incendios para los últimos 136 años (1876-2011), en el bosque de $P$. hartwegii, muestra que de fínales del siglo XIX a la primera década del siglo XXI ha permanecido una frecuencia ininterrumpida del fuego. Sin embargo, posterior a 1988 se detecta un cambio en la frecuencia de incendios, intervalos de 15 y 11 años en las últimas décadas, tendencia que obedece posiblemente a una mayor efectividad en el control de incendios a partir de 1985. Se acepta la primera hipótesis, que plantea que los regímenes del fuego han cambiado en las últimas décadas, cambio asociado a la influencia humana. El cambio de la frecuencia de incendios es una tendencia que aún se puede corregir con buenas prácticas de manejo del fuego; por ello, se recomienda mantener la frecuencia de incendios dentro de la amplitud de su variación histórica.

Se presenta una relación importante entre condiciones de sequía modulada por ENSO y la presencia de incendios. Eventos El Niño que representan condiciones de sequía para el centro de México, están asociados significativamente con años de incendios, estos resultados sustentan la segunda hipótesis de investigación, que establece que condiciones de sequía han influido en la frecuencia histórica del fuego en esta región del centro del país.

\section{AGRADECIMIENTOS}

Esta investigación fue posible gracias al financiamiento SEP-CONACYT a través del proyecto "Estudio de la relación clima-incendios en el norte-centro de México" y al proyecto "Reconstrucción de la frecuencia histórica de incendios y caracterización de las cargas de combustible en ecosistemas forestales del norte-centro de México", con fondos del INIFAP. Gracias a Omar Durán Guerra, Laura Hernández García, Rafael Escobar Cabrera y Gabriel Corona Cuatecontzi por su valioso apoyo durante el trabajo de campo.

\section{REFERENCIAS}

Carrillo-García RL, DA Rodríguez-Trejo, H. Tchikoué, AI Monterroso-Rivas, J Santillan-Pérez. 2012. Análisis espacial de peligro de incendios forestales en Puebla, México. Interciencia 37(9): 678-683.

Cerano-Paredes J, J Villanueva-Díaz, PZ Fulé. 2010. Reconstrucción de incendios y su relación con el clima para la Reserva Cerro El Mohinora, Chihuahua. Revista Mexicana de Ciencias Forestales 1: 63-74.

Cerano-Paredes J, J Villanueva-Díaz, R Cervantes-Martínez, PZ Fulé, L Yocom, G Esquivel-Arriaga, E Jardel-Peláez. 2015. Historia de incendios en un bosque de pino de la sierra de Manantlán, Jalisco, México. Bosque 36(1): 39-50. DOI: 10.4067/S0717-92002015000100004

Cook ER. 2000a. Niño 3 index reconstruction. International TreeRing Data Bank. IGBP PAGES/World Data Center-A for Paleoclimatology Data Contribution Series Number 2000052. NOAA/NGDC Paleoclimatology Program, Boulder, Colorado, USA. Consultado 09 mar. 2015. Disponible en http://www.ncdc.noaa.gov/paleo/recons.html

Cook ER. 2000b. North American drought variability PDSI reconstructions. IGBP PAGES/World Data Center for Paleoclimatology Data Contribution Series Number 2000-074. NOAA/NGDC Paleoclimatology Program, Boulder, Colo- 
rado, USA. Consultado 23 may. 2015. Disponible en http:// www.ncdc.noaa.gov/paleo/recons.html

Dieterich JH, TW Swetnam. 1984. Dendrochronology of a firescarred ponderosa pine. Forest Science 30: 238-247.

Fulé PZ, J Villanueva-Díaz, M Ramos-Gómez. 2005. Fire regime in a conservation reserve, Chihuahua, Mexico. Canadian Journal of Forest Research 35: 320-330. DOI: 10.1139/ X04-173

García ME. 1987. Modificaciones al Sistema de Clasificación Climática de Köppen. México D.F., México. Instituto de Geografía, Universidad Nacional Autónoma de México. Serie Libros Núm. 6. 155 p.

Gergis JL, AM Fowler. 2009. A history of ENSO events since AD 1525: implications for future climate change. Climatic Change 92(3-4): 343-387.

Grissino-Mayer HD, CH Baisan, TW Swetnam. 1994. Fire history and age structure analyses in the mixed conifer and spruce-fir forests of Mount Graham. Final report. Mount Graham Red Squirrel Study Committee. Phoenix, AZ, USA. U.S. Fish and Wildlife Service. 73 p.

Grissino-Mayer HD. 2001. FHX2 - software for analyzing temporal and spatial patterns in fire regimes from tree rings. Tree-Ring Research 57: 115-124.

Heyerdahl EK, E Alvarado. 2003. Influence of climate and land use on historical surface fires in pine-oak forests, Sierra Madre Occidental, Mexico. In Veblen TT, WL Baker, G Montenegro, TW Swetnam eds. Fire and climatic change in temperate ecosystems of the Western Americas. New York, USA. Springer-Verlag. p. 196-217.

Holmes RL. 1983. Computer-assisted quality control in tree ring dating and measurement. Tree-Ring Bulletin 43: 69-75.

IMTA (Instituto Mexicano de Tecnología del Agua, MX). 2009. Extractor Rápido de Información Climatológica III (ERIC), Software. Jiutepec, Morelos, México.

Krawchuk MA, MA Moritz. 2011. Constraints on global fire activity vary across a resource gradient. Ecology 92: 121-132.

Magaña VO, JL Vásquez, JL Pérez, JB Pérez. 2003. Impact of El Niño on precipitation in Mexico. Geofísica Internacional 42: 313-330.

Morales MM. 2005. Programa de manejo forestal para el aprovechamiento persistente de recursos forestales maderables para el tercer ciclo de corta, ejido Rinconada, municipio de Chignahuapan, Puebla. Chignahuapan, Puebla, México. Asesores en Manejo de Recursos Forestales (ASMARF). $107 \mathrm{p}$.

Pausas JG, S Fernández-Múñoz. 2011. Fire regime changes in the Western Mediterranean Basin: from fuel-limited to drought-driven fire regime. Climatic Change 110: 215-226.

Quezada RDA. 2013. Potencial dendrocronológico de Pinus patula (Schl. et Cham.) y reconstrucción de la precipitación para Tlaxcala-Puebla. Tesis de Maestría en Ciencias en Re- cursos Naturales y Medio Ambiente. Bermejillo, Durango, México. Unidad Regional de Zonas Áridas, Universidad Autónoma Chapingo. 80 p.

Rodríguez-Trejo DA. 2001. Ecología del fuego en el ecosistema de Pinus hartwegii Lindl. Revista Chapingo. Serie Ciencias Forestales y del Ambiente 7(2): 145- 151.

Seager R, M Ting, M Davis, M Cane, N Naik, J Nakamura, C Li, E Cook, DW Stahle. 2009. Mexican drought: an observational modeling and tree ring study of variability and climate change. Atmósfera 22:1-31.

Skinner CN, JH Burk, MG Barbour, E Franco-Vizcaíno, SL Stephens. 2008. Influences of climate on fire regimes in montane forests of north-western México. Journal of Biogeography 35: 1436-1451.

Stahle DW, DJ Burnette, J Villanueva-Diaz, RR Heim Jr, FK Fye, J Cerano-Paredes, R Acuña-Soto, MK Cleaveland. 2011. Pacific and Atlantic influences in Mesoamerican over the past millennium. Climate Dynamics 39(6): 1431-1446. DOI 10.1007/s00382-011-1205-z

Stahle DW, ER Cook, DJ Burnette, J Villanueva, J Cerano, JN Burns, RD Griffin, BI Cook, R Acuña, MCA Torbenson, P Sjezner. 2016. The Mexican Drought Atlas: Tree-Ring Reconstructions of the Soil Moisture Balance During the Late Pre-Hispanic, Colonial, and Modern Eras. Quaternary Science Reviews 149: 34-60.

Stephens SL, CN Skinner, SJ Gill. 2003. Dendrochronologybased fire history of Jeffrey pine-mixed conifer forests in the Sierra San Pedro Martir, Mexico. Canadian Journal of Forest Research 33:1090-1101. DOI: 10.1139/X03-031

Stokes MA, TL Smiley. 1968. An introduction to tree-ring dating. Chicago, USA. University of Chicago Press. 73 p.

Swetnam TW, CH Baisan. 2003. Tree-ring reconstructions of fire and climate history in the Sierra Nevada and southwestern United States. In Veblen TT, WL Baker, G Montenegro, TW Swetnam eds. Fire and climatic change in temperate ecosystems of the western Americas. New York, USA. Springer-Verlag. p. 158-195.

Trenberth KE. 1997. The definition of El Niño. Bulletin of the American Meteorological Society 78(12): 2771-2777. DOI: 10.1175/1520-0477

Westerling AL, HG Hidalgo, DR Cayan, TW Swetnam. 2006. Warming and earlier spring increase western US forest wildfire activity. Science 313: 940-943.

Yocom LL, PZ Fulé, PM Brown, J Cerano-Paredes, J VillanuevaDíaz, DA Falk, E Cornejo-Oviedo. 2010. El Niño Southern Oscillation effect on a fire regime in northeastern Mexico has changed over time. Ecology 91(6): 1660-1671.

Yocom LL, PZ Fulé. 2012. Human and climate influences on frequent fire in a high-elevation tropical forest. Journal of Applied Ecology 49(6): 1356-1364. DOI: 10.1111/j.13652664.2012.02216.x 
